\title{
Complicated appendicitis in a COVID -19 positive child: A case report
}

\author{
Sujata Kanhere ${ }^{1}$, Irphan Ali², Varsha Phadke ${ }^{3}$, Sneha Nagpure ${ }^{4}$, Tilakdas Shetty ${ }^{5}$, Nikhil Doke ${ }^{6}$, Asit Patil ${ }^{6}$, \\ Swapnali Mirajkar ${ }^{6}$, Vaibhavi Jaywant ${ }^{6}$ \\ From ${ }^{1}$ Professor and HOD, ${ }^{2}$ Senior Resident, ${ }^{3}$ Professor, ${ }^{4}$ Assistant Professor, ${ }^{6}$ Junior Resident, Department of Pediatrics, ${ }^{5}$ Professor and HOD, \\ Department of Surgery, K.J. Somaiya Medical College, Hospital and Research Centre, Mumbai, Maharashtra, India
}

\begin{abstract}
Appendicectomy is the standard management for acute appendicitis. The pandemic has made us aware of the risks of surgical procedures associated with coronavirus disease 2019 (COVID-19) infection. Here, we present the case of a 5-year-old COVID-positive male child who came with high-grade fever and pain in the abdomen along with vomiting. He was diagnosed to have appendicular perforation with an abscess on abdominal ultrasonography and computerized tomography scan. In view of COVIDs positive status and continued clinical and radiological improvement with intravenous (IV) antibiotics, he was managed conservatively. There was complete resolution of the abscess on follow-up, thereby deferring the need for surgery and thus avoiding complications associated with the procedure. Recent evidence suggests that conservative management with IV antibiotics in selected patients may provide a favorable outcome. This will reduce the morbidity and mortality associated with surgery of a COVID patient. Our case report supports the role of a conservative approach in the management of acute complicated appendicitis in a COVID-positive child.
\end{abstract}

Key words: Appendicitis, Appendicular abscess, Appendicular perforation, Conservative management, COVID -19

A ppendicitis is the most common surgical abdominal emergency seen in the casualty. Common complaints are fever, malaise, anorexia with pain in the abdomen, and vomiting. Diagnosis requires a high level of suspicion as less than $50 \%$ present with classical symptoms [1]. Ultrasonography (USG) and abdominal computerized tomography (CT) help clinch the diagnosis [2]. Delay in the diagnosis can lead to appendiceal perforation, peritonitis, septicemia, and even death. Appendicectomy remains the gold standard in the management of acute appendicitis (AA). Recent reports support a conservative approach in the management of AA with the use of intravenous (IV) antibiotics [3]. Reduced risks of surgery and anesthesia along with a shorter hospital stay and reduced cost of treatment are some other benefits of conservative management of AA [3-5].

Coronavirus disease (COVID)-19 infection presenting with gastrointestinal (GI) symptoms in the pediatric age group has been recently reported in a few studies [3-6]. Tullie et al. have reported a series of eight children presenting at a single center in the UK with COVID-19 infection [6]. Compromised lung function in a COVID-19-positive patient poses a high perioperative risk in addition to cytokine storm causing systemic inflammatory response syndrome and multiple organ dysfunction syndrome.

\section{Access this article online}

Received - 28 December 2020

Initial Review - 12 January 2021

Accepted - 30 January 2021

DOI: $10.32677 /$ IJCR.2021.v07.i02.003
The role of conservative management, particularly in COVID times, needs to be considered. We present the case of a COVID-19 positive child with AA, appendicular perforation, and abscess. The patient was successfully managed conservatively, supporting the role of non-operative management for AA in selected cases.

\section{CASE REPORT}

A 5-year-old male child was brought to the casualty with complaints of high-grade fever, gradually increasing pain in the abdomen in the past 3 days and vomiting since morning on the day of admission. The pain was gradual in onset, dull aching in nature, moderate to severe in intensity, localized over the right iliac fossa, aggravated by food, cough, or on walking, and relieved only after medications. There were four episodes of nonprojectile, non-bilious vomiting on the day of admission since morning. There was decreased oral intake due to vomiting and pain in the abdomen. There was no significant history of any illness or surgery. The patient belonged to a high-risk containment zone for COVID-19 though he had never come in direct contact with any COVID-19-positive patient. He had no symptoms other than fever and GI symptoms suggestive of COVID-19 infection.

Examination of the patient was done after donning personal protective equipment. The child was ill looking but well nourished,

Correspondence to: Dr. Sujata Kanhere, Department of Pediatrics, K.J. Somaiya Medical College, Hospital and Research Centre, Mumbai - 400 022, Maharashtra, India. E-mail: sujatak@somaiya.edu

(C) 2021 Creative Commons Attribution-NonCommercial 4.0 International License (CC BY-NC-ND 4.0). 
febrile with an axillary temperature of $38^{\circ} \mathrm{C}$, and had tachycardia (heart rate 168 beats/min). He was normotensive with a blood pressure of 90/60 documented on a manual sphygmomanometer. His oxygen saturation was $100 \%$ on room air. Per abdomen examination revealed a rigid abdomen with rebound tenderness. Other systemic examination was normal.

$\mathrm{X}$-ray chest was normal and X-ray abdomen did not show any air-fluid level. A CT scan abdomen done after examination and the initial stabilization of the child was suggestive of appendicular perforation (Fig. 1). These X-ray and CT scan findings along with the presenting features and examination findings confirmed the diagnosis of complicated appendicitis.

A surgical opinion was taken and the child was started on IV antibiotics (piperacillin- tazobactam $100 \mathrm{mg} / \mathrm{kg} /$ day in three divided doses, metronidazole $10 \mathrm{mg} / \mathrm{kg} /$ dose 3 times a day, and amikacin $15 \mathrm{mg} / \mathrm{kg} /$ day single dose). Blood investigations were suggestive of leukocytosis and neutrophilia favoring the diagnosis (Table 1). A nasopharyngeal swab was sent for reverse transcriptase polymerase chain reaction (RT-PCR) as per the institutional protocol during the current outbreak. The report was positive for COVID-19 infection. The COVID-positive status of the child put him at high perioperative risk. USG abdomen done after the RT-PCR report was suggestive of appendicular perforation and abscess (Fig. 2a).

The child was clinically and hemodynamically stable with no features of sepsis. After consultation with the surgical team, a decision to observe and continue conservative management was taken. Serial USGs done showed a reduction in the size of the collection of the appendicular abscess. Laboratory reports showed a decrease in leucocyte count (Table 1). He was continued on IV antibiotics for 7 days followed by oral cefixime for 7 days. There was a documented relief in the abdominal pain by day 4 of therapy and the child was started on oral feeds gradually by day 5 of antibiotics. This significant improvement in the general and clinical condition of the child, corroborated on the serial USGs, reduced the need for surgical intervention. He was discharged after 7 days of antibiotics.

After discharge, the patient was quarantined along with his family at a designated COVID center. Follow-up was done for the next 7 days telephonically. One month after the discharge, a follow-up USG abdomen was done which showed complete resolution of the appendicular abscess (Fig. 2b).

\section{DISCUSSION}

COVID-19 infection caused by SARS COVID-2 can have a varied presentation. Angiotensin-converting enzyme-2 receptors are found on the cell membrane of the lungs, intestine, arteries, heart, and kidneys. The COVID-19 virus gains entry into the cells through these receptors $[7,8]$. This could possibly explain the GI symptoms at presentation in a COVID infected child. Viral shedding per rectally has also been reported in some studies with a positive rectal RT-PCR swab [9]. This may also suggest a possibility of viral transmission by a feco-oral route along with the known transmission through droplets [10]. GI symptoms of

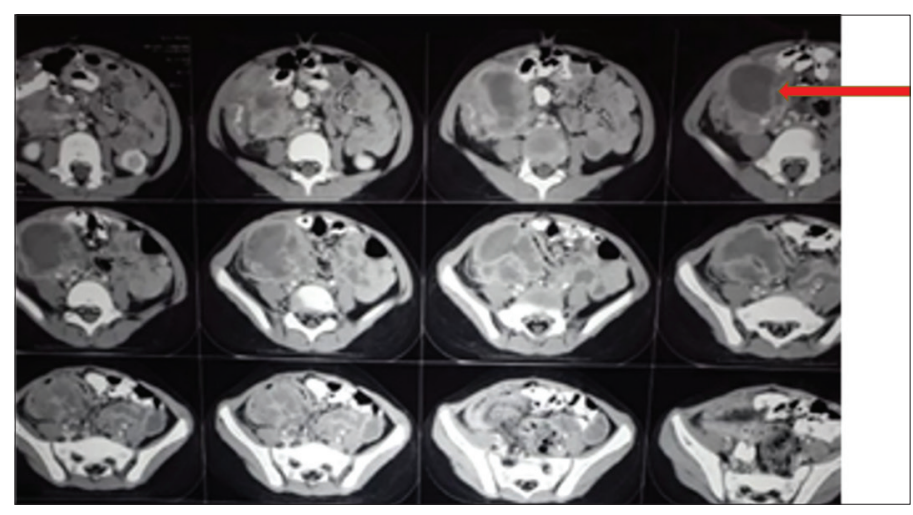

Figure 1: Computed tomography scan abdomen demonstrating appendicular perforation

COVID-19 include anorexia, abdominal pain, vomiting, and diarrhea which may be the sole presenting feature [11-14].

Even though AA is a common surgical condition seen in children, the diagnosis needs a high degree of suspicion due to atypical presentations and overlapping features with other GI diseases like gastroenteritis. Parental interpretation of symptoms and difficult clinical examination in a child makes diagnosis a challenge [15]. As a result, delay in diagnosis may be observed in as high as $57 \%$ of children younger than 6 years of age [4] with a proportionally rising risk of appendicular perforation [5].

Our patient had a clinical history suggestive of AA. Progression of symptoms over 3 days and a rigid abdomen on examination gave rise to suspicion of appendicular perforation. Surgical intervention was deferred as the child was positive for COVID-19 infection. A few randomized control studies done in adults with uncomplicated AA treated conservatively have been published during this pandemic with a success rate of approximately $65-90 \%[16,17]$. Similar studies in a small number of pediatric patients indicate that conservative management may be safe [18-20]. A 13-year-old girl with uncomplicated appendicitis managed conservatively with IV antibiotics has been reported [21]. However, another 4-year-old girl with complicated appendicitis with COVID-19 was reported recently but was managed by open appendectomy [22].

We had to be extra cautious knowing that life-threatening complications such as peritonitis and sepsis can occur if the abscess is not drained. The visibly improving clinical, laboratory, hematological, and radiological parameters led to continuing the conservative approach. The complete resolution of the abscess after 1 month indicated the effectiveness of the conservative approach in selected patients.

In the COVID-19 pandemic, management protocols have changed due to the risk of aerosol-generating surgical and anesthesia procedures. Although studies indicate that adult patients with appendicitis have been treated conservatively during this pandemic, randomized control trials (RCT) done have not supported any specific antibiotic and patients responded well to a variety of antibiotics. Not all patients can be managed conservatively and may need surgical intervention. Svensson et al. did a pilot RCT with 50 children diagnosed for acute non-perforated appendicitis where 28 children were managed by surgical intervention and 22 (44\%) 
Table 1: Investigations done at admission and during course of hospitalization

\begin{tabular}{lcccccccc}
\hline Date & Hemoglobin & WBC count & Platelet & Neutrophil (\%) & Creatinine & Blood urea nitrogen & Serum ferritin & D-dimer \\
\hline May 23, 20 & $10.8 \mathrm{~g} / \mathrm{dl}$ & $20,600 / \mathrm{mm}^{3}$ & 5 lakhs & 79 & $0.4 \mathrm{mg} / \mathrm{dl}$ & $8.8 \mathrm{mg} / \mathrm{dl}$ & \\
May 26, 20 & $10.9 \mathrm{~g} / \mathrm{dl}$ & $9000 / \mathrm{mm}^{3}$ & 6.5 lakh & 46 & $0.4 \mathrm{mg} / \mathrm{dl}$ & $4.6 \mathrm{mg} / \mathrm{dl}$ & $63 \mathrm{ng} / \mathrm{ml}$ & $268 \mathrm{mcg} / \mathrm{ml}$ \\
\hline
\end{tabular}
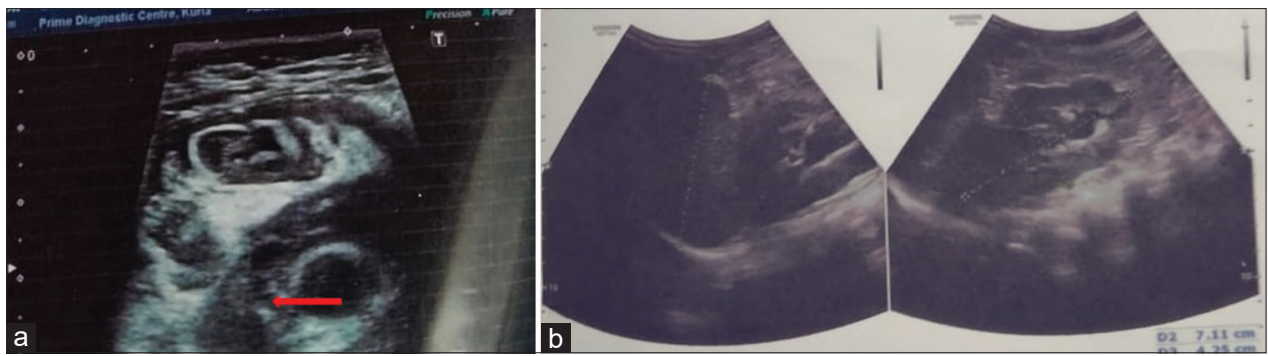

Figure 2: Ultrasonography abdomen (a) $24 \mathrm{~h}$ after admission showing appendicular abscess; (b) follow-up after 1 month showing complete resolution of abscess

children were managed conservatively [18]. However, not many case reports or series have been published in the literature, using the same approach, in complicated appendicitis. We have successfully managed a case of COVID-19-positive child with AA along with appendicular perforation and abscess without surgery.

\section{CONCLUSION}

It is possible to successfully manage a COVID-19 positive child with AA with appendicular perforation and abscess without surgical intervention. However, surgical management of AA continues to be the gold standard and should be considered if a patient does not respond to conservative management. In the COVID-19 pandemic, in view of increased surgical and anesthetic risk, the role of conservative management with appropriate IV antibiotics and strict monitoring is worth considering.

\section{REFERENCES}

1. Society of American Gastrointestinal and Endoscopic Surgeons: Pediatric Appendicitis. SAGES. Wikimedia Foundation; 2013. https://www.sages. org/wiki/pediatric-appendicitis. [Last accessed on 2020 Jun 16].

2. Pade KH. Point-of-care ultrasound facilitates bedside diagnosis of appendicitis with an appendicolith in a pediatric patient. Pediatr Emerg Care 2018;34:818-9.

3. Hansson J, Körner U, Khorram-Manesh A, Solberg A, Lundholm K. Randomized clinical trial of antibiotic therapy versus appendicectomy as primary treatment of acute appendicitis in unselected patients. Br J Surg 2009;96:473-81

4. Coccolini F, Fugazzola P, Sartelli M, Enrico C, Maria GS, Gioacchino L, et al. Conservative treatment of acute appendicitis. Acta Biomed 2018;89:119-34.

5. Hansson J, Körner U, Ludwigs K, Johnsson E, Jönsson C, Lundholm K. Antibiotics as first-line therapy for acute appendicitis: Evidence for a change in clinical practice. World J Surg 2012;36:2028-36.

6. Tullie L, Ford K, Bisharat M, Watson T, Thakkar H, Mullassery D, et al. Gastrointestinal features in children with COVID 19: An observation of varied presentation in eight children. Lancet 2020;4:e19-20.

7. Zhou P, Yang XL, Wang XG, Hu B, Zhang L, Zhang W, et al. A pneumonia outbreak associated with a new coronavirus of probable bat origin. Nature 2020;579:270-3.

8. Hamming I, Timens W, Bulthuis ML, Lely AT, Navis GJ, Goor HV. Tissue distribution of ACE2 protein, the functional receptor for SARS coronavirus. A first step in understanding SARS pathogenesis. J Pathol 2004;203:631-7.
9. Xu Y, Li X, Zhu B, Liang H, Fang C, Gong Y, et al. Characteristics of pediatric SARS-CoV-2 infection and potential evidence for persistent fecal viral shedding. Nat Med 2020;26:502-5.

10. Liu Y, Ning Z, Chen Y, Guo M, Liu Y, Gali NK, et al. Aerodynamic characteristics and RNA concentration of SARS-CoV-2 aerosol in Wuhan hospitals during COVID-19 outbreak. Nature 2020;582:557-60.

11. Tian Y, Rong L, Nian W, He Y. Review article: gastrointestinal features in COVID-19 and the possibility of faecal transmission. Aliment Pharmacol 2020;51:843-51.

12. Ong J, Young BE, Ong S. COVID-19 in gastroenterology: A clinical perspective. Gut 2020;69:1144-5.

13. Jin X, Lian JS, Hu JH, Gao J, Zheng L, Zhang YM, et al. Epidemiological, clinical and virological characteristics of 74 cases of coronavirus-infected disease 2019 (COVID-19) with gastrointestinal symptoms. Gut 2020;69:1002-9.

14. Gu J, Han B, Wang J. COVID-19: Gastrointestinal manifestations and potential fecal-oral transmission. Gastroenterology 2020;158:1518-9.

15. Wang ZH, Ye J, Wang YS, Liu Y. Diagnostic accuracy of pediatric atypical appendicitis: Three case reports. Medicine (Baltimore) 2019;98:15006.

16. Varadhan KK, Humes DJ, Neal KR, Lobo DN. Antibiotic therapy versus appendectomy for acute appendicitis: A meta-analysis. World J Surg 2010;34:199-209.

17. Wilms IM, de Hoog DE, de Visser DC, Janzing HM. Appendectomy versus antibiotic treatment foracute appendicitis. Cochrane Database Syst Rev 2011;11:CD008359.

18. Svensson JF, Patkova B,Almstrom M, Naji H, Hall NJ, Eaton S, et al. Nonoperative treatment with antibiotics versus surgery for acute nonperforated appendicitis in children: A pilot randomized controlled trial. Ann Surg 2015;261:67-71.

19. Tanaka Y, Uchida H, Kawashima H, Fujiogi M, Takazawa S, Deie K, et al. Long-term outcomes of operative versus nonoperative treatment for uncomplicated appendicitis. J Pediatr Surg 2015;50:1893-7.

20. Minneci PC, Mahida JB, Lodwick DL, Sulkowski JP, Nacion KM, Cooper JN, et al. Effectiveness of patient choice in nonoperative vs surgical management of pediatric uncomplicated acute appendicitis. JAMA Surg 2016;151:408-15.

21. Jones BA, Slater BJ. Non-operative management of acute appendicitis in a pediatric patient with concomitant COVID-19 infection. J Pediatr Surg Case Rep 2020;59:101512.

22. Alsuwailem A B, Turkistani R, Alomari M. Complicated appendicitis in a pediatric patient with COVID-19: A case report. Cureus 2020;12:e8677.

Funding: None; Conflicts of Interest: None Stated.

How to cite this article: Kanhere S, Ali I, Phadke V, Nagpure S, Shetty T, Doke N, Patil A, Mirajkar S, Jaywant V. Complicated appendicitis in a coronavirus disease-19-positive child. Indian J Case Reports. 2021;7(2):50-52. 